Marion UHLIG, «Le cheval de la langue : héroïsme et art d'écrire dans Galeran de Bretagne de Renaut », dans Y. HAMEL et M. BOUCHARD (dir.), Portrait de l'homme de lettres en héros, @ nalyses, hiver 2006

\title{
Marion UHLIG
}

\section{Le cheval de la langue : héroïsme et art d'écrire dans Galeran de Bretagne de Renaut}

Orationis autem ipsius tamquam armorum est uel ad usum comminatio et quasi petitio uel ad uenustatem ipsa tractatio $^{1}$ (1978, IX, I, 33).

À la suite des Anciens, les romanciers du Moyen Âge aiment à rappeler qu'à travers la voix du héros, c'est celle du poète qui se fait entendre. Parmi les procédés référentiels courants de la littérature médiévale, il en est un, majeur, qui n'a de cesse d'assimiler l'art de la guerre à l'art oratoire ou, pour employer des termes chers aux trouvères, la chevalerie à la clergie. Le texte médiéval, en vertu de sa conscience aiguë de la dimension textuelle des œuvres, fait ainsi de l'exploit chevaleresque le miroir des arts d'écrire. Une telle pratique connaît une fortune considérable à partir du $\mathrm{XII}^{\mathrm{e}}$ siècle, notamment grâce à l'essor que prend la dialectique au sein des sept arts libéraux ${ }^{2}$. À l'âge du symbole, cette science belliqueuse emprunte en effet, dans les textes de fiction, la noble image de la chevalerie.

C'est a fortiori le cas dans le roman de Galeran de Bretagne où, en l'absence de prologue, les actions des personnages reflètent la poétique de l'œuvre. Mutilé en son commencement, l'unique exemplaire — il s'agit du manuscrit 24042 du Fonds français de la Bibliothèque nationale de France - nous prive de renseignement sur les desseins du trouvère Renaut. Or Roger Dragonetti, dans son étude sur Galeran de Bretagne (p. 229-260), a montré comment les prouesses hérö̈ques des personnages palliaient ce manque, délibéré ou accidentel, en

\footnotetext{
${ }_{1}$ « Les figures de mots servent comme des armes pour la menace et, en quelque sorte, pour l'assaut, mais on les manie aussi pour la grâce seule. » (trad. de J. Cousin)

2 Les sept artes sont la grammaire, la dialectique, la rhétorique (le trivium) et l'arithmétique, la géométrie, l'astronomie et la musique (le quadrivium).
} 
Marion UHLIG, «Le cheval de la langue : héroïsme et art d'écrire dans Galeran de Bretagne de Renaut », dans Y. HAMEL et M. BOUCHARD (dir.), Portrait de l'homme de lettres en héros, @nalyses, hiver 2006

proposant le reflet des arts rhétoriques qui sous-tendent la création romanesque.

Ce roman matrimonial du début du $\mathrm{XIII}^{\mathrm{e}}$ siècle exploite la trame narrative du Lai du Freisne de Marie de France et l'émaille d'épisodes empruntés au Conte de Floire et Blancheflor, attribué à Robert d'Orbigny. Comme le lai, le roman débute par la calomnie publique d'une châtelaine. Jalouse de sa voisine, jeune mère de beaux jumeaux, dame Gente l'accuse de fréquenter deux hommes à la fois. Lorsqu'à son tour, elle donne naissance à des jumelles, elle se trouve contrainte d'exposer l'une d'elles pour échapper au déshonneur. Abandonnée dans le feuillage d'un frêne qui lui donne son nom, la jeune enfant est recueillie par l'abbesse de Beauséjour et élevée aux côtés de son neveu, le jeune seigneur Galeran. Le roman quitte alors le modèle de Marie de France pour se rapprocher de celui de Floire et Blancheflor. On reconnaîtra en effet dans le schème narratif de ces enfances amoureuses l'influence du roman idyllique médiéval. La suite du récit, plus proche du roman d'apprentissage, puise à des sources narratives diverses pour rapporter la séparation des amants, leur quête solitaire puis leurs retrouvailles. Galeran s'éloigne de Fresne, encouragé par son entourage à devenir chevalier, puis à trouver une épouse de noble lignage. Au terme de maintes errances, il se résout à épouser Florie, l'autre jumelle, en raison de sa ressemblance avec l'aimée. Quant à l'héroïne, qui parvient à gagner sa vie grâce au travail de ses mains, elle se fait reconnaittre de son amant, juste avant les noces, en jouant sur sa harpe le chant de leur amour. Le roman renoue avec le lai dans l'épisode final où la mère, qui identifie en Fresne sa propre fille grâce à une étoffe brodée, interrompt le mariage et unit les amants.

Dans ce roman fondé sur la réécriture, trois épisodes constituent des innovations. La composition et l'exécution par les amants d'un lai qui enclot le secret de leur amour, l'apprentissage de la mesure, dévolu à Galeran, dans l'épreuve des armes et des charmes, et enfin la couture d'une robe permettant à Fresne de retrouver son ami et sa famille, ne ressortissent pas aux intertextes que nous avons mentionnés. Or, un bref regard sur ces aventures originales suffit à constater qu'elles revêtent au sein du récit une fonction précise : il s'agit, dans ce roman 
Marion UHLIG, «Le cheval de la langue : héroïsme et art d'écrire dans Galeran de Bretagne de Renaut », dans Y. HAMEL et M. BOUCHARD (dir.), Portrait de l'homme de lettres en héros, @nalyses, hiver 2006

né de l'agrégat de sources diverses, de réfléchir sur la composition romanesque. À ce titre, les actions des héros, dans les trois passages, incarnent chacune à leur façon une facette du processus d'écriture. Ainsi, l'auteur ne met plus en scène les figures de la langue à partir de l'unique force masculine, mais également à partir de la force féminine. Dans ce redoublement, les exploits de l'aiguille sont investis de la même vocation proprement rhétorique que la prouesse de l'épée. Alors, lorsque la réflexivité intervient au sein d'un roman d'amour, qu'en est-il de la représentation du clerc en guerrier? En quoi la figuration du couple amoureux, plutôt que celle du chevalier, influence-t-elle l'analogie entre l'écrivain et le héros? Quelle est la conséquence de tels remaniements dans la conception médiévale du roman matrimonial? Pour répondre à ces questions, nous examinerons le traitement réservé dans le récit aux motifs traditionnels de la musique, de la chevalerie et du tissage.

\section{L'amour du roman}

Le roman de Galeran de Bretagne répond aux 518 vers du Lai du Freisne par une amplificatio de près de 8000 octosyllabes qui consacre un large espace aux amours des deux héros. Si, chez Marie de France, l'idylle du couple n'occupait qu'un seul vers (v. 313), notre roman fait en revanche de l'éveil sentimental de Fresne et de Galeran le lieu nodal de l'intrigue. Dans un premier temps, c'est sans doute au Conte de Floire et Blancheflor que l'épisode qui décrit ces amours enfantines puise son inspiration. La naissance presque simultanée des enfants, leur troublante ressemblance et leur éducation commune dans l'abbaye rappellent de manière frappante ce récit byzantin du $\mathrm{XII}^{\mathrm{e}}$ siècle. Le drap de Fresne, brodé par sa propre mère, n'arbore-t-il pas en outre sur son premier quartier les aventures de Floire et de la belle Blanchefleur, «tissues par merveilleux art» (v. 518)? En guise d'hommage à ces amants-jumeaux, scènes et descriptions, individualisées et traitées en alternance, s'enchaînent et multiplient les effets de symétrie spéculaire. Cette succession, qui parait mimer le lien gémellaire, souligne l'identité de ces amoureux qui se ressemblent et se confondent. La description égalitaire des enfants débouche en toute logique sur l'affirmation de leur affection mutuelle. C'est en effet au 
Marion UHLIG, «Le cheval de la langue : héroïsme et art d'écrire dans Galeran de Bretagne de Renaut », dans Y. HAMEL et M. BOUCHARD (dir.), Portrait de l'homme de lettres en héros, @nalyses, hiver 2006

cœur de cette paire symbiotique que l'amour éclot. Toutefois, l'expression des sentiments quitte sans tarder le modèle fusionnel et l'indistinction caractéristiques des émois juvéniles pour s'engager sur la voie de l'énamoration courtoise. À la faveur d'images symboliques qui s'inscrivent dans la tradition de l'amour-souffrance, Galeran et Fresne sont identifiés à de fins amants. Obsédés par la pensée de l'être aimé, ils subissent alors les affres du désir. Comme Hélène et Pâris, dont la destinée est inscrite sur le second quartier du drap brodé, comme Tristan et Iseut, auxquels nos héros se comparent souvent, ils perdent alors l'appétit et le sommeil, pâlissent et dépérissent à vue d'œil :

Amours les detient et les lace, Ja leur detaille le mengier, Ja viennent a tres grant dangier, Ja mes ne leur chault qu'i soit aise; Paine et travail, pour qu'i leur plaise, Sçavent souffrir et endurer; Ja ont changé ris pour plourer. (v. 1342-1348)3

Mais le recours à une casuistique amoureuse fondée sur les expressions consacrées de la lyrique courtoise n'est que transitoire. Si le poète détaille les phases de l'évolution qui transforme les enfantsfleurs en amants courtois, il ajoute bientôt une donnée supplémentaire : la chasteté. Les amours adultères, dont l'exemple a pour un temps séduit les héros, se trouvent dès lors condamnés par le conteur. Fresne et Galeran renoncent à l'enlèvement et à l'adultère décrits par le lai, mais aussi par les sources brodées sur le drap, pour inscrire la relation amoureuse dans la distance et la parole. Le roman moralise ici ses modèles et annonce l'exigence de virginité. Tel est le sens des « chastoiements » - enseignements — que le bon chapelain Lohier adresse tour à tour aux deux amants. Ces leçons soulignent

\footnotetext{
${ }^{3}$ Amour les tient prisonniers et les lie, Il leur ôte la faim,

Ils sont maintenant sous une emprise très puissante.

Il leur importe peu de se sentir bien;

Ils savent maintenant supporter et endurer

La peine et la souffrance, pour plaire à Amour.

Ils ont désormais échangé le rire pour les larmes. (Je traduis)
} 
Marion UHLIG, «Le cheval de la langue : héroïsme et art d'écrire dans Galeran de Bretagne de Renaut », dans Y. HAMEL et M. BOUCHARD (dir.), Portrait de l'homme de lettres en héros, @nalyses, hiver 2006

l'importance de la tempérance et donnent une inflexion morale à la passion des héros. Encouragés par Lohier, Fresne et Galeran sont conduits à dire leur amour et à nommer l'élu de leur cœur. À défaut d'unir leur corps, tous deux doivent trouver les mots susceptibles de signifier leur passion. Comme pour témoigner de la nécessité de cette réorientation éthique et langagière, l'expression du sentiment quitte les canons rhétoriques de la lyrique courtoise; les formules d'obédience ovidienne laissent place à la peinture d'un amour civilisateur et dispensateur de vertu. La disparition des symptômes physiques de la souffrance amoureuse scelle la transformation : après avoir confessé leurs sentiments, les amants retrouvent le bonheur. Pâleur et tristesse disparaissent au profit des chants, des rires et, surtout, des aveux réciproques.

À la faveur de tels sermons, le programme narratif se dessine avec netteté : il s'agit de proposer l'exemple d'un amour chaste et égalitaire, orienté vers des fins matrimoniales. Une doxa de la passion conforme aux préceptes religieux naît ainsi du jeu intertextuel. Mais l'insistance du poète à décrire les phases successives de cette élaboration amoureuse recèle un enjeu supplémentaire. Au Moyen Âge, la littérature repose sur un principe d'imitation et d'émulation selon lequel chaque œuvre convoque ses sources pour mieux s'en démarquer. ${ }^{4}$ Or, en puisant tour à tour aux modèles consacrés de la passion romanesque, l'amour dépeint dans le roman renvoie l'image de cette technique de composition. Ainsi, la conception sentimentale qui s'échafaude dans Galeran de Bretagne semble refléter le processus d'écriture. Cette vocation réflexive prêtée au thème de l'amour s'accentue dans la scène finale de l'épisode lorsque les amants, chantant ensemble le Lay Galeren le Breton (v. 1982), fondent une analogie entre amour et création poétique.

Cette dernière scène de la séquence narrative dédiée aux amours naissantes des héros a fonction de paradigme. Le matin de mai où, parés de leurs plus beaux atours, ceux-ci se retrouvent pour déclarer

\footnotetext{
${ }^{4}$ Matthieu de Vendôme, comme les autres théoriciens médiévaux des arts poétiques, souligne la nécessité de compléter ce qui a été dit de manière insuffisante dans le modèle, de changer ce qui sonne mal et d'abolir ce qui est superflu (p. 180).
} 
Marion UHLIG, «Le cheval de la langue : héroïsme et art d'écrire dans Galeran de Bretagne de Renaut », dans Y. HAMEl et M. BOUCHARD (dir.), Portrait de l'homme de lettres en héros, @nalyses, hiver 2006

leur amour, fixe dans leur mémoire comme dans celle du lecteur l'image d'un bonheur inaltérable. Converti en locus amoenus, espace consacré du bonheur romanesque, le verger de Beauséjour prête son décor à la communion des amants. En attendant l'union matrimoniale — et charnelle —, comment trouver meilleur cadre à l'échange d'aveux que ce lieu rhétorique où les amants se joignent dans l'entrelacs des rimes? Hiératiques, vêtus comme pour une cérémonie royale, ils semblent mimer un hymen sacré. Tel est d'ailleurs l'enjeu de la scène : il s'agit de figer dans le récit l'idéal amoureux que les héros auront pour tâche de réaliser. Cet instant de rêve où Fresne et Galeran échangent un double aveu dans le verger figure en effet leur union anticipée dans la langue. À défaut d'abriter les premiers émois érotiques, le verger tient lieu de scène solennelle sur laquelle, par la suite, les pensées reviendront sans cesse. Or, parmi les serments échangés, c'est le lai lyrique, don de l'amant à sa promise, qui cristallise la joie du moment. Galeran a composé un lai dont Fresne, pour prolonger l'échange dans la musique, transpose les accords sur sa harpe :

«Fresne, fait il, j'ay esprouvé

Mon engin a un novel lay,

Si desir moult que sans delay

Tout le deduit vous en apreigne.

Mais comment que il vous en preigne,

$\mathrm{Ne}$ vueil qu'autre que vous le sache;

Mieulx vouldroie estre d'une hache

Occis qu'autruy l'apreïssiez. » (v. 2278-2285) ${ }^{5}$

Il ne fait aucun doute que, lorsque la jeune fille écoute avec ravissement les tendres paroles et fait sonner sur son instrument les

\footnotetext{
${ }^{5}$ «Frêne, dit-il, je me suis ingénié

À composer un nouveau lai,

Et je désire plus que tout que, sans attendre,

Je puisse vous en apprendre tout le plaisir.

Mais, quoi qu'il arrive,

Je ne veux pas qu'un autre que vous le connaisse;

Je préférerais être tué à coups de hache

Plutôt que vous ne l'appreniez à quelqu'un d'autre. » (Je traduis)
} 
Marion UHLIG, «Le cheval de la langue : héroïsme et art d'écrire dans Galeran de Bretagne de Renaut », dans Y. HAMEL et M. BOUCHARD (dir.), Portrait de l'homme de lettres en héros, @ nalyses, hiver 2006

notes entendues, la plénitude acoustique métaphorise le plaisir d'aimer. Le « deduit» (v. 2214, 2277 et 2281), mot-clef de la lyrique courtoise, naît alors de l'exécution commune du chant d'amour. L'accord à l'unisson du chant et de la harpe immortalise ainsi le souvenir du bonheur partagé. En aval, Fresne et Galeran tendront à la réalisation effective de cette alliance sonore perçue comme en songe. Et le roman, de même, n'aura de cesse de concrétiser ce serment matrimonial qui, placé à l'orée du roman, fait figure de programme narratif.

Par sa vocation proleptique, le lai incarne la source même du récit. Le titre du roman, qui renvoie au nom du lai, le prouve ${ }^{6}$. On pourrait penser qu'un intitulé tel que Galeran de Bretagne prend le contre-pied d'un récit largement propice aux personnages féminins. Mais il n'en est rien. Dissimulée sous l'archaïsme du nom masculin, la nouveauté du roman réside précisément dans l'interaction des deux héros, immortalisée par la scène du lai. Et c'est de cette fusion harmonieuse entre les genres dont le roman témoigne lorsqu'il arbore le nom du lai. Partant, l'équation formée par le couple devient le paradigme diégétique de la création poétique. En mettant en scène sa propre genèse au travers des vœux des jeunes amants, le roman célèbre l'union équilibrée du couple.

La suite ne sera que la mise en application de ce programme narratif et musical à partir duquel le roman s'auto-engendre. C'est là la fonction des quêtes individuelles accomplies par les amants. Absentes du lai de Marie de France, les métaphores de la chevalerie et du tissage incarnent, au gré des épreuves du couple d'amis, les péripéties d'une écriture empreinte d'une réflexion sur sa propre composition.

\section{Une chevalerie du discours}

Deux catastrophes successives mettent fin à la période ensoleillée des amours enfantines. La première a lieu à la Saint-Jean, lorsqu'un messager porteur de mauvaises nouvelles parvient à Beauséjour. Les parents de Galeran sont morts, et le jeune homme doit se rendre en

\footnotetext{
${ }^{6}$ Sur le titre du roman, voir Michèle Gally (p. 95). L'explicit du manuscrit unique comporte «Cy finist le livre de Galeren, conte de Bretaigne ».
} 
Marion UHLIG, «Le cheval de la langue : héroïsme et art d'écrire dans Galeran de Bretagne de Renaut », dans Y. HAMEL et M. BOUCHARD (dir.), Portrait de l'homme de lettres en héros, @ nalyses, hiver 2006

Angleterre pour recevoir ses fiefs et entrer en chevalerie (v. 26882697). Fresne et Galeran prennent congé dans la peine. Malgré les visites, bientôt interdites, puis les lettres, les liens des amants se distendent au fil du temps.

Lorsque le héros prête hommage au roi d'Angleterre et reçoit le serment d'allégeance de ses propres barons, la chevalerie fait une entrée inattendue au cœur du roman d'amour. La quête héroïque revêt l'aspect d'un apprentissage militaire; Galeran doit acquérir gloire et renommée. Dès lors, pour le héros, c'est par la conscience du devoir à remplir que le sentiment s'exprime. L'amour, en effet, doit être mérité par de hauts faits d'armes, selon la règle du code chevaleresque: "L'homme noble qui veut aimer doit se distinguer par ses prouesses » (v. 3082-3083), lui signifie son maitre. Londres, Nantes, Metz et la Roche-Guyon seront les étapes de cette initiation au terme de laquelle le héros deviendra un parfait chevalier. Parmi les domaines concernés par son apprentissage, l'équitation est un art qu'il doit maîtriser. Bon écuyer, Galeran fait preuve d'aisance et de mesure lorsqu'il soumet son cheval à la discipline : «Le Breton [Galeran] sait mesurer son cheval» (v. 4876), dit le texte. La mesure, voilà prononcé un maitre mot! N'estelle pas l'apanage du prud'homme, garant de l'harmonie et du maintien de la société féodale? Les traités moraux du Moyen Âge la mentionnent en bonne place parmi les qualités chevaleresques qui assurent la permanence de la courtoisie ${ }^{7}$. "Cortesia non es als mas mesura" ", dit le troubadour Folquet de Marseille (XII, v. 41). Or, dans notre roman, la mesure chevaleresque dont Galeran fait la preuve semble concerner les arts du bien-dire; c'est ce que suggère le passage où le héros prépare la diatribe qu'il destine au duc de Lorraine :

Or li convendra recevoir

Sens et pourveance et mesure

Par quoy li homs en hounour dure;

Car chascum estuet mesurer, Se il veult en hounour durer,

\footnotetext{
${ }^{7}$ Brunet Latin (II, 74). On trouve un passage identique chez le Pseudo-Guillaume de Conches (p. 142).

${ }^{8}$ « La courtoisie, ce n'est rien d'autre que de la mesure » (Je traduis).
} 
Marion UHLIG, «Le cheval de la langue : héroïsme et art d'écrire dans Galeran de Bretagne de Renaut », dans Y. HAMEL et M. BOUCHARD (dir.), Portrait de l'homme de lettres en héros, @nalyses, hiver 2006

Le chief avant de son affaire, Et puis aprés la fin pourtraire, Et mectre ensemble fin et chief, Qu'en son affaire n'ait meschief. (v. 3454-3462) ${ }^{9}$

Les parties du discours, comme la succession des arguments, ont été étudiées avec soin et revêtent la parole d'une parfaite maîtrise. À tel point que l'affaire de Galeran, comme le révèle Roger Dragonetti, semble se confondre dans ce passage avec l'affaire du narrateur, qui médite son roman et en équilibre l'ensemble (p. 240).

En faisant de la «doulce alaine » (v. 1193) du héros l'équivalent de sa maitrise de l'équitation, le roman aborde la question de l'éloquence dans son rapport avec la chevalerie ${ }^{10}$. Renaut fait ainsi des chevaliers consommés, qui accomplissent des prouesses dans l'art du dressage et savent modérer leur propos, les détenteurs de l'excellence. L'écart de langage, à l'inverse, s'assimile à la perte de contrôle du destrier. Aussi la mauvaise parole prononcée au début du roman place-t-elle la mère de Fresne à l'écart de cette pratique alliant aisance oratoire et maîtrise chevaleresque :

Car el faisoit trop tost aller

Le cheval de sa langue e courre,

Que nulz ne lui povoit rescourre

Quant elle estoit en haulte alaine. (v. 36-39) ${ }^{11}$

\footnotetext{
${ }^{9}$ Il devra maintenant recevoir

Le sens, la prévoyance et la mesure

Grâce auxquels l'homme est maintenu dans l'honneur;

En effet, chacun doit mesurer

- S'il souhaite conserver son honneur -

La première partie de son discours,

Et ensuite en reproduire la fin,

Puis assembler la fin et le début,

Pour qu'il n'y ait pas d'imperfections dans son discours. (Je traduis)

${ }^{10}$ Cf. Marion Uhlig.

${ }^{11}$ Elle faisait bien trop vite aller

Et courir le cheval de sa langue,

De telle sorte que nul ne pouvait l'empêcher

Lorsqu'elle était en haute haleine. (Je traduis)
} 
Marion UHLIG, «Le cheval de la langue : héroïsme et art d'écrire dans Galeran de Bretagne de Renaut », dans Y. HAMEL et M. BOUCHARD (dir.), Portrait de l'homme de lettres en héros, @ nalyses, hiver 2006

Le même sort est réservé à l'abbesse Ermine qui, mise au courant des amours secrètes de son neveu et de l'orpheline recueillie sur le frêne, calomnie la pauvre enfant:

Or voit de quel pié Fresne cloche

La dame qui son cheval broche;

Ire la transporte et ardure,

Sa langue broche oultre mesure

Qui li desvoie le courage. (v. 3913-3917) ${ }^{12}$

La chevauchée, en vertu de la densité métaphorique du vocabulaire, se soumet à l'expression de la (dé)mesure langagière. Or tout porte à croire que, ce faisant, le roman place les qualités héroïques du côté masculin. Il situe en effet son ouvre dans le noble champ de cette chevalerie d'armes et de lettres dont parlent le Roman de la rose (v. 1144211443) ou le prologue du Roman de Thèbes (v. 13-16) ${ }^{13}$, qui associe l'excellence du guerrier à celle du clerc. Si le rythme et la mesure distinguent les preux des lâches et des médisants, ils dissocient également, semble-t-il, les hommes des femmes.

Toutefois, si Galeran prouve sa valeur par une maitrise chevaleresque qui fait écho au rythme de l'écriture, un long parcours sera encore nécessaire au jeune homme avant de montrer le même savoir-faire dans les arts d'aimer. L'éloquence masculine montre ici ses limites. Lorsque le semblant - l'apparence, le portrait — de Fresne, perdue depuis plusieurs années, prend vie en la personne de Florie, l'illusion est si forte que le héros s'y perd. Héritière digne du jeune seigneur breton, la jumelle de Fresne porte les traits de l'aimée. Face à un leurre aussi parfait, rien d'étonnant à ce que naisse dans le cœur du héros, qui

\footnotetext{
${ }^{12}$ La dame qui éperonne son cheval

Voit maintenant de quel pied Frêne boite;

La colère l'emporte et l'enflamme,

Elle éperonne outre mesure sa langue,

Qui lui fait perdre la raison. (Je traduis)

${ }^{13}$ La chevauchée est aussi le lieu consacré de l'inventio; selon la rêverie poétique de Guillaume de Poitiers, le premier troubadour, dont le poème « fut trouvé en dormant / Sur un cheval... », le pas régulier de la monture imprègne de son rythme la chanson lyrique (p. 92, v. 5-6).
} 
Marion UHLIG, «Le cheval de la langue : héroïsme et art d'écrire dans Galeran de Bretagne de Renaut », dans Y. HAMEL et M. BOUCHARD (dir.), Portrait de l'homme de lettres en héros, @nalyses, hiver 2006

croit Fresne morte, le sentiment de l'original retrouvé. Galeran choisit alors d'aimer la jumelle comme s'il s'agissait de Fresne. Mais s'il croit d'abord goûter au bonheur regagné devant ce simulacre de la femme aimée, force lui est bientôt de constater que Florie n'est que pure apparence, pure «semblance» de l'aimée (v. 6442). À la veille de ses noces, il constate la vacuité de la copie qu'il avait prise pour le modèle lui-même :

«Sont, fait il, desvé my amy,

Qui me vont mariant a force?

Donner m'en pueent bien l'escorce

Et li fuz dessouz en soit leur.

$[\ldots]$

C'est Flourie, qui a la chiere

Et le semblant plus avenant

Qu'elle n'ait tout le remenant;

Li remenant voir m'en descorde,

Puis qu'au semblant point ne s'acorde;

Moult y amasse l'acordance,

Mais, puis que j'y voy la doubtance,

Je ne m'acort point a li prendre. » (v. 6820-6833) ${ }^{14}$

Au contact de la doublure de Fresne, si ressemblante soit-elle, le héros ressent avec acuité la soif d'authenticité. Il regrette alors l'absence de l'aimée, seule figure authentique qui, annihilant la distance entre le modèle et son portrait, possédait le «remenant», ce reste qui

\footnotetext{
14 «Mes amis sont-ils devenus fous, dit-il,

Eux qui me forcent à me marier?

Ils peuvent bien me donner l'écorce

Mais le bois, en dessous, ne m'appartient pas.

[...]

C'est Florie, dont le visage

Et l'apparence sont bien plus charmants

Que ne l'est le reste (l'intérieur);

En vérité, je refuse le reste

Puisqu'il ne s'accorde pas à l'apparence;

J'aurais beaucoup aimé que ces principes concordent

Mais, puisque ce n'est pas le cas,

Je ne puis me résoudre à la prendre pour épouse. » (Je traduis)
} 
Marion UHLIG, «Le cheval de la langue : héroïsme et art d'écrire dans Galeran de Bretagne de Renaut », dans Y. HAMEL et M. BOUCHARD (dir.), Portrait de l'homme de lettres en héros, @ nalyses, hiver 2006

permettait la communion véritable des amants. Le souvenir du chant d'amour à l'unisson rappelle à Galeran que les deux voix ne s'élèvent plus: la voix de Fresne, comme sa harpe, manquent. Privé des charmes musicaux qui magnifiaient ses talents oratoires, Galeran s'égare. Éloquence et mesure ne lui sont plus d'aucune utilité, car il manque l'accompagnement musical, composante essentielle du pacte d'amour, dont Fresne seule est la détentrice.

En envisageant la ressemblance de Florie avec Fresne selon un rapport de la copie à son modèle, le roman met en scène une réflexion sur l'écriture $^{15}$. Le poète recourt en effet au motif folklorique de la gémellité pour se livrer à une critique de la reproduction. En ce sens, la confrontation du héros avec le substitut imparfait de l'aimée illustre l'impossibilité de saisir une matière dans sa surface, sans en considérer l'intériorité. Or il en est ainsi de la composition d'un récit: elle ne ressortit pas à une pure imitation, mais à une pratique qui exige une part d'invention.

\section{Textus}

Au centre du roman, la dispute de Fresne et de l'abbesse occasionne une seconde crise qui fait pendant à la disparition des parents de Galeran. Dame Hermine, qui craint la mésalliance de son neveu, accuse Fresne de dévergondage et la chasse de l'abbaye. Prenant soin d'emporter sa harpe et les objets légués à sa naissance - le précieux oreiller en plume de phénix et le drap brodé - la jeune fille change de nom et d'origine. Sous le nom de Mahaut la Bretonne (v. 4188), elle erre sur les routes de France et, déguisée en ménestrel, harpe et récite des contes pour gagner sa vie. Il s'agit d'une nouvelle naissance pour l'enfant trouvée. À Rouen, elle rencontre une famille d'adoption et mène auprès de dame Blanche et de sa fille Rose une existence nouvelle. L'amitié de Rose lui permet alors de renouer avec le savoirfaire, chère à sa mère, des ouvrières aux belles mains :

A Rose donne mainte guimple

\footnotetext{
${ }^{15}$ Voir Michèle Gally (p. 101).
} 
Marion UHLIG, «Le cheval de la langue : héroïsme et art d'écrire dans Galeran de Bretagne de Renaut », dans Y. HAMEL et M. BOUCHARD (dir.), Portrait de l'homme de lettres en héros, @nalyses, hiver 2006

Et maint tyssu et mainte atache;

De son mestier veult qu'elle sache,

Or l'en aprent, si fait que gente :

Tant en retient et met s'entente

Que bien euvre de soie et d'or,

Dont elle assemble bon tresor. (v. 4278-4284) ${ }^{16}$

Or, c'est précisément dans cet art vertueux des brodeuses que Mahaut, après plusieurs années d'absence et d'oubli, puisera l'énergie nécessaire à sa résurrection en Fresne.

À la nouvelle du mariage de Galeran et de Florie, notre hérö̈ne décide de se rendre en compagnie de Rose à la fête, sans manquer d'emporter avec elle son drap précieux. Parvenue à la Roche-Guyon, lieu de la noce, Fresne transforme le drap illustré en une robe magnifique (v. 6744-6756). Sans détruire les fragments, elle prend soin de conserver intactes les images brodées, faisant du legs maternel son propre vêtement, de telle sorte que «l'œuvre y apparaît comme auparavant» (v. 6757). Dans cette transformation de l'objet par l'habile couturière, le message dévié des amours légendaires contenu dans les images originelles se trouve recomposé. Réaménageant les images, la coupe et l'ajustement du drap produisent une unité nouvelle. Cette réinvention de la parure illustrée en une œuvre parfaite et entière fait de la création féminine le double symbolique de l'écriture $^{17}$. Comment ne pas songer à l'association, chère aux rhétoriciens antiques et médiévaux, entre le vêtement et le texte? Le travail de l'ornement, de la broderie, désigne aussi l'ouvrage de réinvention de la langue dans la Poetria Nova de Geoffroi de Vinsauf, auteur d'un art poétique médiolatin (p. 221). À la faveur d'une telle

\footnotetext{
${ }^{16}$ Elle donne à Rose nombre de guimpes,

De tissus et de liens.

Elle souhaite qu'elle connaisse son métier,

Alors elle le lui enseigne et agit en femme avisée :

Rose retient si bien les leçons et se donne tant de peine

Qu'elle brode fort bien la soie et l'or

Et assemble ses confections en un beau trésor. (Je traduis)

${ }^{17}$ Sur le caractère autoréférentiel que la tradition médiévale prête au motif de la broderie à la faveur de l'étymologie commune du texte et du tissu, voir Romaine Wolf-Bonvin.
} 
Marion UHLIG, «Le cheval de la langue : héroïsme et art d'écrire dans Galeran de Bretagne de Renaut », dans Y. HAMEL et M. BOUCHARD (dir.), Portrait de l'homme de lettres en héros, @ nalyses, hiver 2006

analogie, le drap remanié par Fresne devient l'image même du travail poétique. Derrière l'héroïne, qui modifie l'ouvrage de Gente — ou œuvre-mère —, on distingue le profil du poète, déconstruisant la source bretonne pour enrichir son roman. En vertu de ces métaphores textiles, la robe de Fresne incarne un art d'écrire qui, dans une littérature basée sur l'imitation, quête la novitas dans l'émulation.

Or, en investissant ce rapprochement entre la brodeuse et la figure du poète, c'est aussi son côté féminin que le roman affirme. La fonction dévolue aux héros — incarner une idée formelle — n'est plus l'apanage de l'homme, dans Galeran de Bretagne. Tandis que l'aventure chevaleresque se déroule au loin, la femme enrichit de son art les desseins du textus. Nul doute que la broderie, ainsi mise en rapport avec les arts poétiques, fait l'originalité de ce roman longtemps mal reçu par la critique, qui n'y voyait qu'un «tissu d'emprunts cousus bout à bout» (1930, p. 212). Tissage et chevalerie revêtent alors dans l'espace du texte des fonctions équivalentes; c'est de leur combinaison que naît le roman. Mais il faut attendre la fin du récit pour que résonne à nouveau l'accord à l'unisson des voix féminine et masculine. Tandis que Galeran, constatant son égarement, se trouve prêt à renoncer à l'illusion, Fresne lui apparaît avant les noces pour réaliser l'unité à laquelle tous deux aspirent. Or, le traitement du thème gémellaire contraint à varier la scène de reconnaissance topique : ni le visage, ni le corps ne suffisent à identifier Fresne, car ils sont dépourvus de sens. C'est donc par les attributs qui lui sont propres que la jeune fille, revêtue de sa nouvelle robe, mais le visage dissimulé sous une guimpe, se fait connaittre. Les talents de Fresne, exaltés au cours du récit, atteignent dans ce passage une fonction dramatique exemplaire: ils incarnent le remenant, le reste susceptible de distinguer la jumelle désirée de son double fallacieux. Galeran authentifie son amie à l'écoute du lai d'amour qu'elle entonne. Par ce chant de leur enfance, le lai Galeran, Fresne restitue l'harmonie qui manquait à l'intégrité du héros. Car, comme celui-ci le précise au vers 6831, c'est en elle que réside «l'acordance » (v. 6831). Seule la voix, que le portrait ne pouvait reproduire, permet à l'amant d'identifier Fresne sans la voir. Il ne manque plus aux retrouvailles des amants qu'une légitimation sociale. Elle sera fournie par la mère dans l'épisode final du roman, qui est à 
Marion UHLIG, «Le cheval de la langue : héroïsme et art d'écrire dans Galeran de Bretagne de Renaut », dans Y. HAMEl et M. BOUCHARD (dir.), Portrait de l'homme de lettres en héros, @nalyses, hiver 2006

nouveau proche du Lai du Freisne. Dame Gente, qui reconnait l'enfant abandonnée à la vue du drap devenu robe, restitue à la jeune fille rang et parenté. Florie se retire au couvent, tandis que sont célébrées les noces de Fresne et de Galeran.

$\mathrm{Au}$ terme de notre enquête, l'indépendance de Galeran de Bretagne par rapport à ses sources apparaît de façon plus nette. Sous la plume de Renaut, le thème populaire de la femme éprouvée dans son dévouement amoureux, développé par le lai de Marie de France, devient un plaidoyer éloquent en faveur de la complémentarité des principes féminin et masculin. Certes, les emprunts au roman idyllique, tout comme l'investissement original du motif gémellaire, contribuent à cette transformation des personnages et de la situation réciproque des deux héros. Mais c'est davantage l'identification du poète à un modèle non plus exclusivement masculin, mais fondé sur l'équilibre des genres, qui constitue le gain majeur de la réécriture. Si la conception sentimentale exposée dans le roman se construit sur les poncifs de la littérature amoureuse, elle s'en émancipe bientôt pour déboucher sur une réflexion rhétorique et esthétique. Renaut fait du couple le paradigme de la création littéraire et renouvelle, partant, la conception médiévale de l'héroïsme autant qu'il repense la logique du roman matrimonial. En soulignant la prééminence de l'invention sur l'imitation, il pose un regard nouveau sur l'histoire d'amour et sur les liens entre homme et femme.

\section{Bibliographie}

Brunet Latin. 1975 [1948], Li Livres dou tresor, éd. critique de F. J. Carmody, Los Angeles, Berkeley University Press.

Dragonet'TI, Roger. 1987, Le Mirage des sources. L'art du faux dans le roman médiéval, Paris, Seuil.

GALLY, Michèle. 2000, "Ouvrages de dames. L'invention romanesque au XIII siècle ", Revue des Langues Romanes: le roman médiéval, la fiction réaliste au XII siècle, vol. 104, no 1, p. 91-110. 
Marion UHLIG, «Le cheval de la langue : héroïsme et art d'écrire dans Galeran de Bretagne de Renaut », dans Y. HAMEL et M. BOUCHARD (dir.), Portrait de l'homme de lettres en héros, @ nalyses, hiver 2006

GeofFroi De Vinsauf. 1924a, Poetria Nova, dans Edmond Faral, Les Arts poétiques du XIIe et du XIIIe siècle : recherches et documents sur la technique littéraire du Moyen Âge, Paris, Champion, p. 202-225.

Guillaume IX D’Aquitaine. 1973, Poesie, éd. critique N. Pasero, Modène, STEM-Mucchi.

HoepfFner, Ernest. 1930, «Les Lais de Marie de France dans Galeran de Bretagne et Guillaume de Dole», Romania, n 56, p. 196-231.

JeAn De Meun. 1966a, Le Roman de la rose, éd. de F. Lecoy, Paris, Champion.

JEAN RENART. 1975 [1925], Galeran de Bretagne. Roman d'aventure du XIII siècle, éd. de L. Foulet, Paris, Champion.

Marie de FranCE. 1966b, Lais, éd. de J. Rychner, Paris, Champion.

MAtTHIEU DE Vendôme. 1924b, Ars versificatoria, dans Edmond Faral, Les Arts poétiques du XII et du XIII siècle : recherches et documents sur la technique littéraire du Moyen Âge, Paris, Champion, p. 178-195.

Pseudo-Guillaume DE Conches. 1929, Das Moralium dogma philosophorum, Lateinisch, Altfranzösisch und Mittelniederfränkisch, herausg. J. Holmberg, Uppsala, Almqvist \& Wiksells.

QuintiLIEN. 1978, Institution oratoire, éd. et trad. de J. Cousin, Paris, Les Belles Lettres, coll. «Universités de France ».

Roman De ThÈbes (Le). 1966d, éd. de G. Raynaud de Lage, Paris, Champion.

Stronski, Stanislaw. 1968 [1910], Le Troubadour Folquet de Marseille, Genève, Slatkine Reprints. 
Marion UHLIG, «Le cheval de la langue : héroïsme et art d'écrire dans Galeran de Bretagne de Renaut », dans Y. HAMEL et M. BOUCHARD (dir.), Portrait de l'homme de lettres en héros, @nalyses, hiver 2006

UhLig, Marion. À paraitre en 2005, "Pour ce que cuers ne puet mentir : le personnage maternel dans Galeran de Bretagne de Renaut », in Keith Busby et Chris Kleinhenz (eds), Selected Proceedings du Congrès international de littérature courtoise, Madison.

Wolf-Bonvin, Romaine. 1998, Textus. De la tradition latine à l'esthétique du roman médiéval, Paris, Champion. 